\title{
Si Substrate-Based Metamaterials for Ultrabroadband Perfect Absorption in Visible Regime
}

\author{
Qi Han, ${ }^{1}$ Lei Jin, ${ }^{1}$ Yongqi Fu, ${ }^{1}$ and Weixing $\mathrm{Yu}^{2,3}$ \\ ${ }^{1}$ School of Physical Electronics, University of Electronic Science and Technology of China, Chengdu 610054, China \\ ${ }^{2}$ State Key Laboratory of Applied Optics, Changchun Institute of Optics, Fine Mechanics and Physics, Chinese Academy of Sciences, \\ Changchun, Jilin 130033, China \\ ${ }^{3}$ Institute of Micro \& Nano Optics, College of Optoelectronic Engineering, Shenzhen University, Shenzhen 518060, China
}

Correspondence should be addressed to Qi Han; hanqi1115@163.com

Received 8 July 2014; Accepted 11 September 2014; Published 23 December 2014

Academic Editor: Fengzhou Fang

Copyright (C) 2014 Qi Han et al. This is an open access article distributed under the Creative Commons Attribution License, which permits unrestricted use, distribution, and reproduction in any medium, provided the original work is properly cited.

We report the broadband efficient light absorbing property of a structure of quadrangular frustum pyramid array in visible regime. The structure can absorb light efficiently with an average absorptivity of 0.98 over the whole visible waveband. In addition, it is found that this kind of super light absorbing can maintain an average of 0.9 for a wide incident angle range. The perfect absorbing property of the metamaterial-based nanoring array is attributed to the effect of the Fabry-Perot resonance. The structure is possible to be used as a type of Si photonics devices in future photonic circuits.

\section{Introduction}

With the decrease of fossil fuel and the constant aggravation of pollution, solar energy is regarded as one of the most plenty sources of renewable energy and much effort has been made to develop highly efficient photovoltaic devices. Nanostructured photovoltaics promise simultaneously to increase the efficiency and decrease the cost of solar cells. Recently, various nanostructures including nanopillar, nanowire, nanocone, and nanohole arrays have been proven possessing unique optical and electronic characteristics for light absorbing and harvesting [1-10]. Tseng et al. demonstrated the enhancing light absorption property of Au nanorings due to their strong localized surface plasmon resonance [11]. Sturmberg et al. showed modal analysis of enhanced absorption in silicon nanowire arrays [12]. These reports laid an academic foundation of optical absorption. Metamaterialbased structures have gained increasingly interest for the last decade due to their unique and extraordinary optical properties. Cui et al. recently reported a one-dimensional metamaterial-based saw-tooth shape absorber and it shows good absorptive property in middle infrared waveband [13]. More recently, a two-dimensional pyramidal shape metamaterial based absorber was developed, which shows the surprisingly high absorptive property in an extraordinary broad waveband ranging from near infrared to long infrared [14]. Most recently, a two-dimensional subwavelength metananopillar array was reported that can achieve an extraordinary absorption in visible waveband [15]. In addition, it was reported that the perfect absorption materials are useful for being used as refractory materials [16].

However, these structures are difficult to be produced in industry due to the complicated nanostructures and materials issue. Current solar cells are $\mathrm{Si}$ substrate-based absorption structure. The incorporation of nanostructures in silicon photovoltaics is of particular importance as these currently make up over $80 \%$ of the photovoltaic market due to silicon's low cost, natural abundance, and compatibility with mature fabrication techniques. Considering this, Si substrate-based structure is an important issue for designing photovoltaic structures. Dühring and Sigmund reported that Si-based nanowires are better than metals ( $\mathrm{Al}, \mathrm{Ag}$, and $\mathrm{Au}$ ) for optical absorption in visible regime [17]. We created a new Si substrate-based metamaterial which has ultrabroad waveband with high absorption in visible regime. Unique features of this proposed metamaterial structure and 


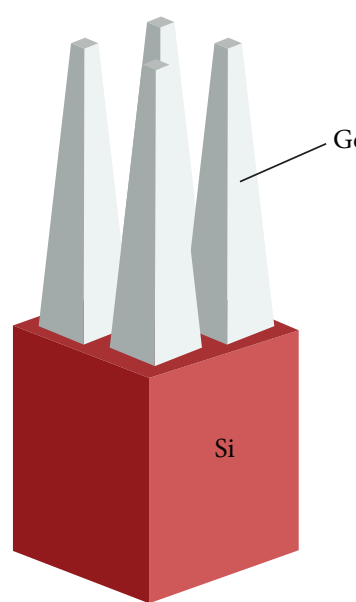

(a)

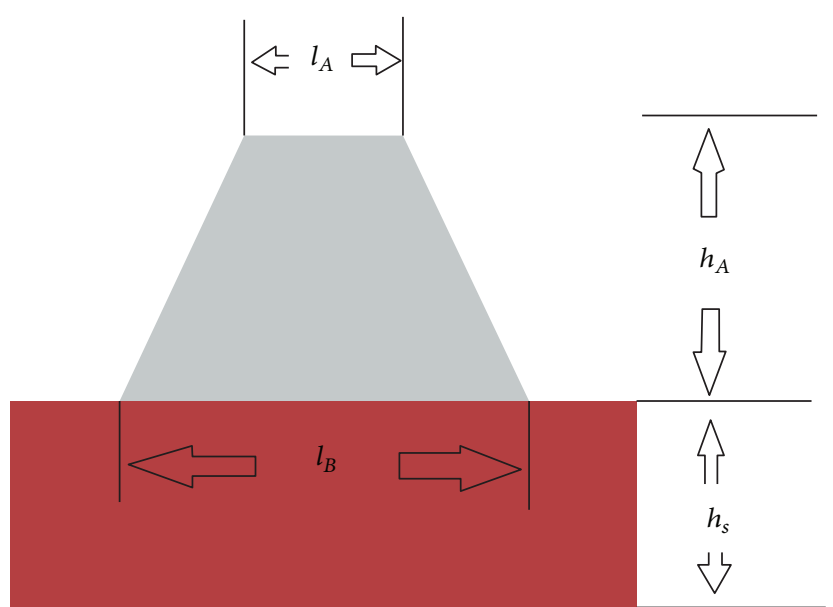

(b)

Figure 1: (a) Schematic diagram of quadrangular frustum pyramid absorber; (b) cross-sectional view of a single quadrangular frustum pyramid.

the corresponding physical mechanism of the light absorbing are presented and explained in below sections.

\section{Computational Setup and Design}

A schematic diagram of the proposed quadrangular frustum pyramid array is shown in Figure 1(a). As can be seen from Figure 1(b), the structure with quadrangular frustum pyramid array is a two-dimensional periodic structure atop a Si thin film $h_{s}=700 \mathrm{~nm}$ in thickness. For the quadrangular frustum pyramid array, its material is Ge. Germanium and silicon belong to indirect-gap materials, but germanium has lower band gap, resulting in photons with lower energy that can excite electrons from valence band to conduction band. It means that germanium material-based structures can increase optical absorptivity in broader band than silicon material. In the meanwhile, with the same energy photons excitation, more heat can be produced; higher temperature and higher photoelectric conversion efficiency are obtained in germanium structure. The top surface is square in which the side length is $l_{A}=50 \mathrm{~nm}$; the bottom surface is also square with side length of $h_{B}=130 \mathrm{~nm}$. A single quadrangular frustum pyramid is $h_{A}=900 \mathrm{~nm}$ in height. Filling factor of the pyramid is 0.4425 . It means that a single quadrangular frustum pyramid is constructed on a Si substrate which has side length of $200 \mathrm{~nm}$.

By employing finite-difference and time-domain (FDTD) algorithm, the absorptive spectrum of the quadrangular frustum pyramid array working in visible regime was calculated, as shown in Figure 2. As can be seen, the average absorptivity of the quadrangular frustum pyramid array in the whole visible waveband reaches as high as 0.98 and has surpassed those solar energy absorbers reported before [1-15]. Then we discuss influence of several parameters while others are fixed. Figure 2(b) shows the influence of Si substrate on absorptivity. It can be seen that with increasing of thickness, the average absorptivity increases as well. That means the deeper the Si substrate is, the better the absorptivity it can reach. For the large refractive index contrast between $\mathrm{Ge}$ and air, electromagnetic field can be coupled efficiently into the quadrangular frustum pyramids at Fabry-Perot resonances, resulting in a significant light-trapping ability boost. For quadrangular frustum pyramid array with small filling factor, a part of the incident light cannot be coupled into quadrangular frustum pyramids but is absorbed in the substrate through resonances.

\section{Results and Analysis}

Prolonging the path of light can provide more times at FabryPerot resonances. That is to say, the longer the light path is, the higher the optical absorptivity will be. Figure 2(c) describes the influence of the height of Ge quadrangular frustum pyramid on absorptivity. It is found that the taller the quadrangular frustum pyramid is, the better the absorptivity it can reach.

Figures 2(d)-2(e) display the influence of the length of top and bottom surfaces on absorptivity, respectively. As can be seen, the best association is that the length of the top and bottom surfaces is $50 \mathrm{~nm}$ and $110 \mathrm{~nm}$, respectively. The base angle of the quadrangular frustum pyramid is $71.56^{\circ}$. Figure 2 shows the superiorities of this structure. These parameters can be changed in a wide range, but the absorptivity can be kept in a similar high level. This is the reason that the structure can allow mechanical errors, and it consists of a $\mathrm{Si}$ substrate and a Ge quadrangular frustum pyramid array. It is much easier than the other reported structures [13-15] for fabrication.

To fully characterize the absorptivity of the quadrangular frustum pyramid array, the relations between the absorptivity and the wide waveband and the incident angle of incoming light were calculated, respectively. Figure $2(\mathrm{f})$ shows the absorptive spectrum in the waveband ranging from $200 \mathrm{~nm}$ to $1000 \mathrm{~nm}$. It is shown that although absorptivity drops 

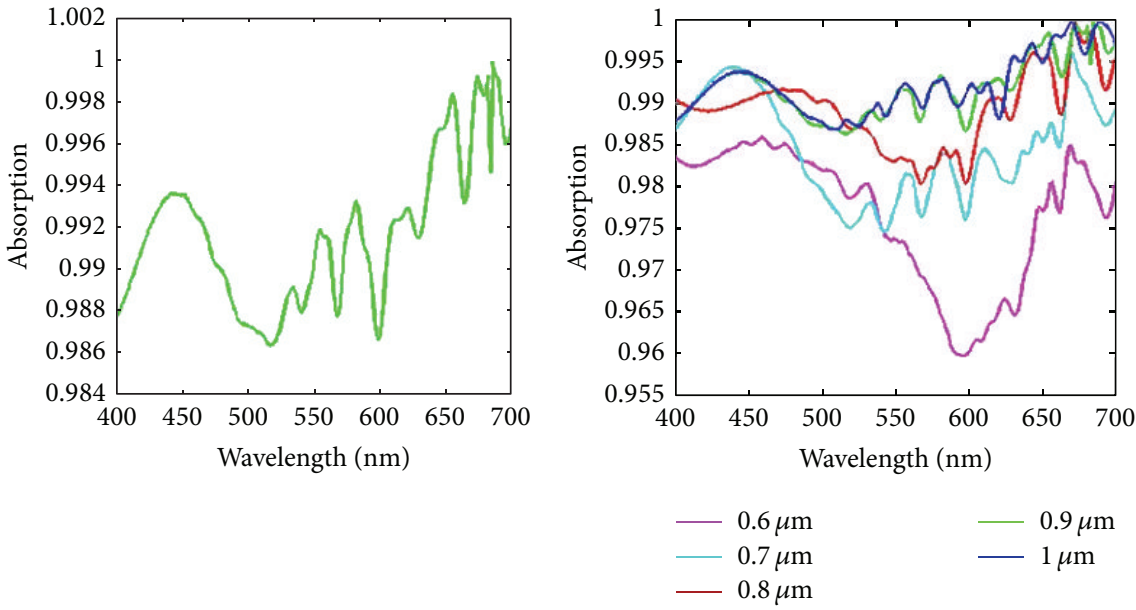

(a)
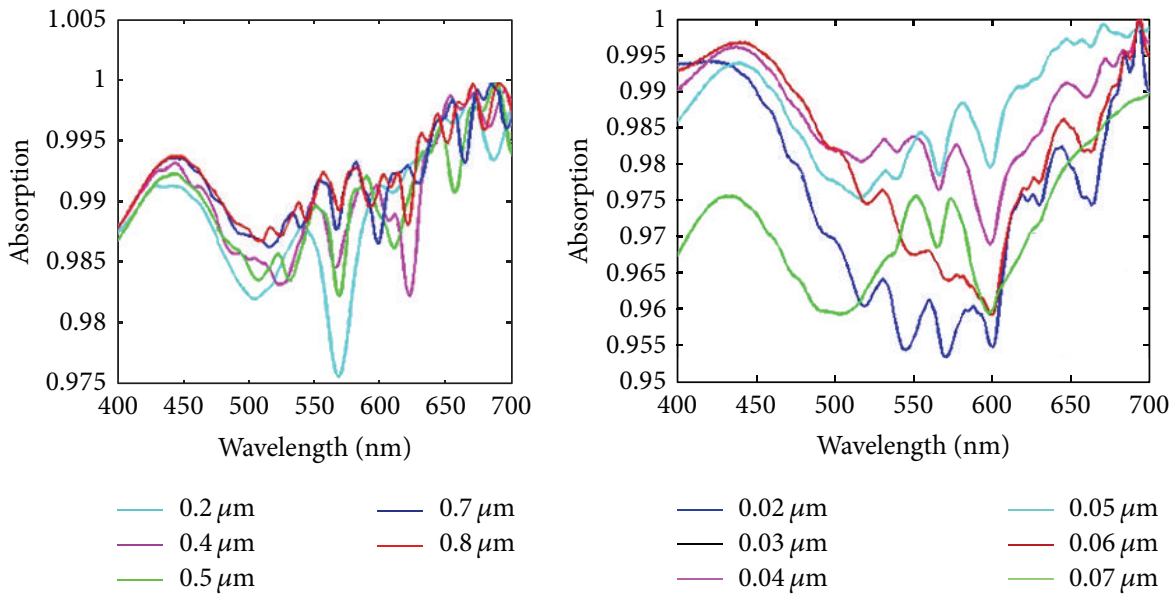

(c)

(d)

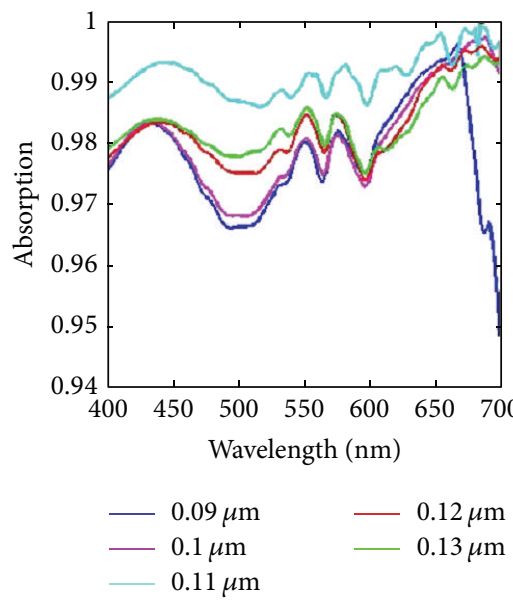

(e)

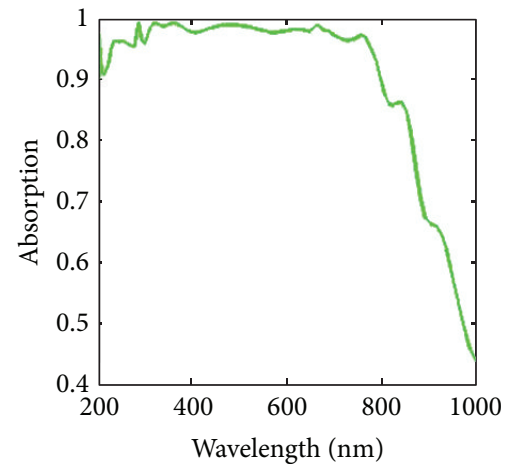

(f)

FIGURE 2: Absorption spectra of the quadrangular frustum pyramid array under normal incident light for various parameters. (a) Under the optimal conditions of $l_{A}=50 \mathrm{~nm}, l_{B}=130 \mathrm{~nm}, h_{A}=0.9 \mu \mathrm{m}$, and $h_{s}=0.7 \mu \mathrm{m}$. (b) For the different height $h_{A}$, when $l_{A}=50 \mathrm{~nm}, l_{B}=130 \mathrm{~nm}$, and $h_{s}=0.7 \mu \mathrm{m}$. (c) For the different thickness $h_{s}$, when $l_{A}=50 \mathrm{~nm}, l_{B}=130 \mathrm{~nm}, h_{A}=0.9 \mu \mathrm{m}$ (d) For the different length $l_{A}$, when $l_{B}=130 \mathrm{~nm}, h_{A}=0.9 \mu \mathrm{m}$, and $h_{s}=0.7 \mu \mathrm{m}$. (e) For the different length $l_{B}$, when $l_{A}=50 \mathrm{~nm}, h_{A}=0.9 \mu \mathrm{m}$, and $h_{s}=0.7 \mu \mathrm{m}$. (f) Absorption spectra of the quadrangular frustum pyramid array with waveband from $200 \mathrm{~nm}$ to $1000 \mathrm{~nm}$. 

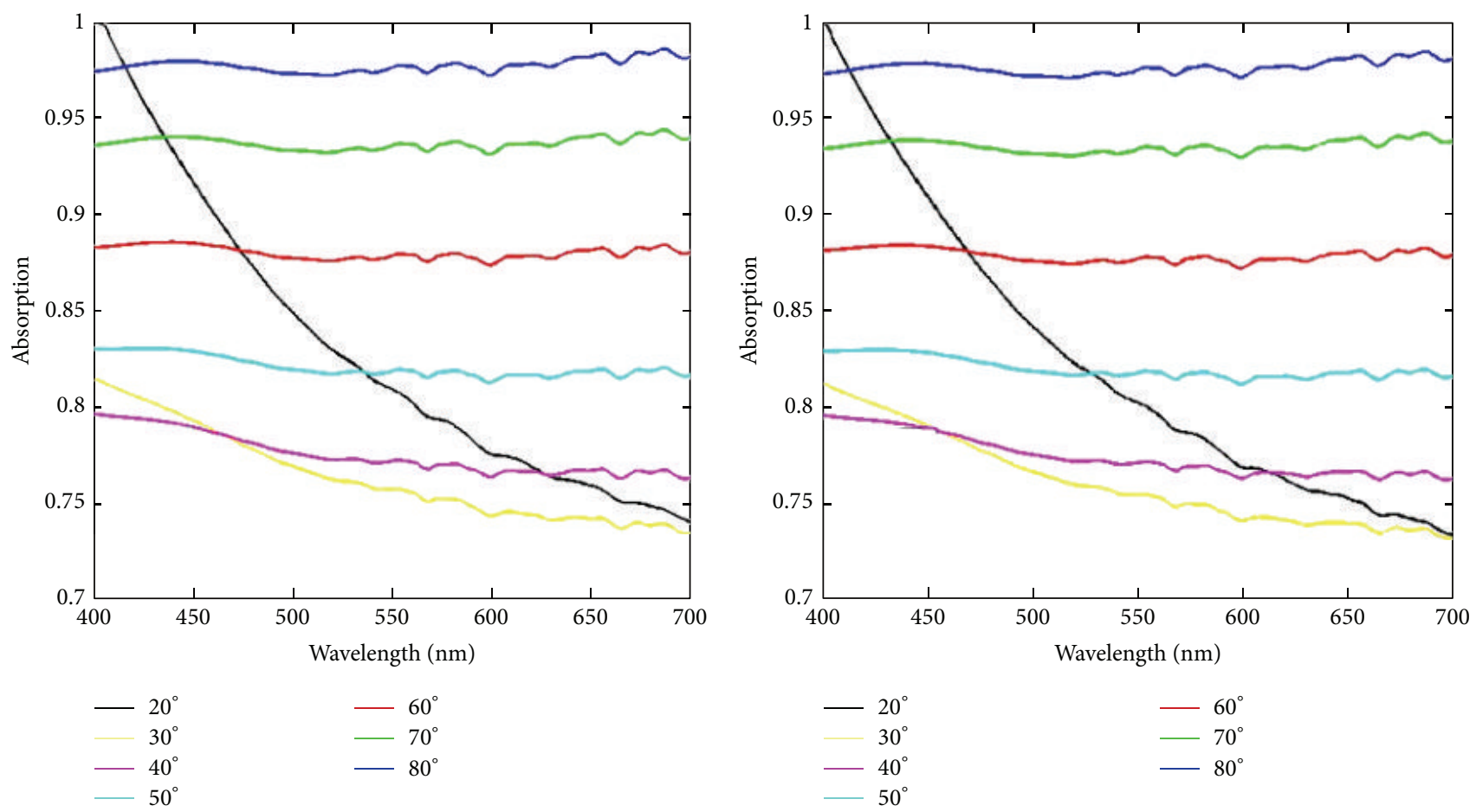

(a)

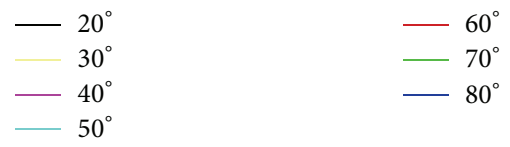

(b)

FIGURE 3: Absorptive spectra of quadrangular frustum pyramid array under various angle. (a) For different incident angles from $20^{\circ}$ to $80^{\circ}$. (b) For different incident angles from $20^{\circ}$ to $80^{\circ}$.

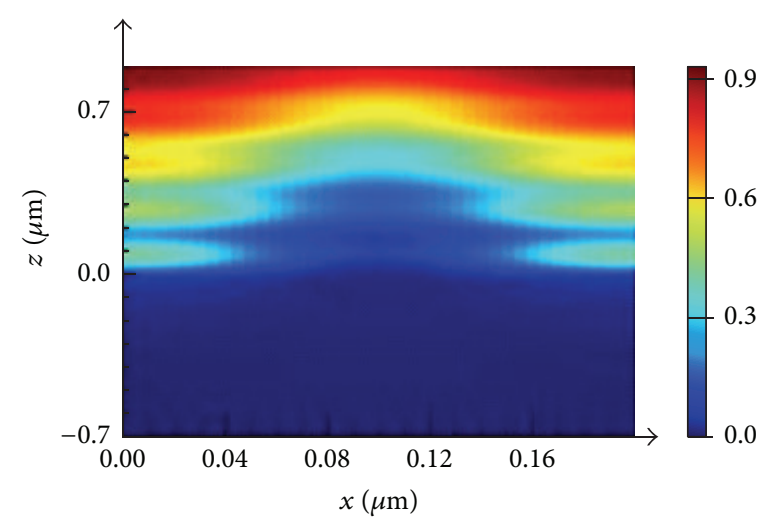

(a)

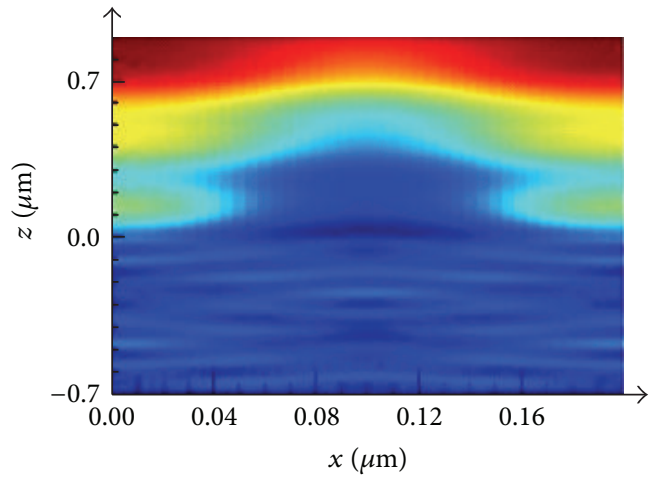

(c)

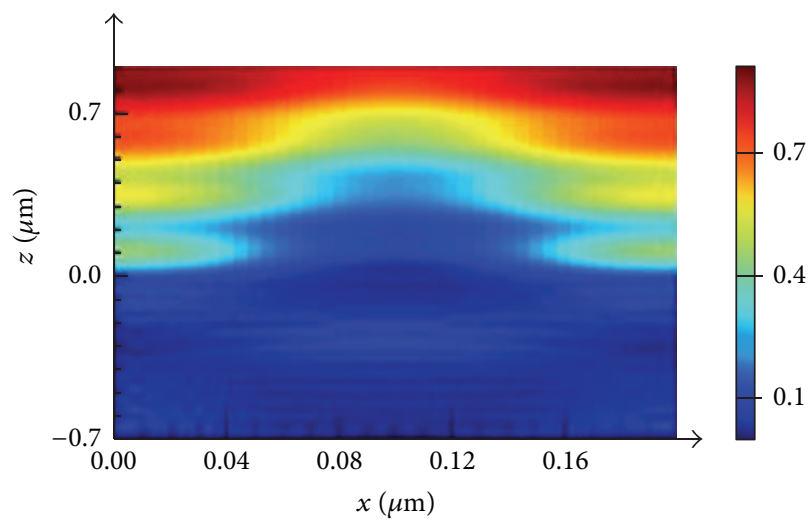

(b)

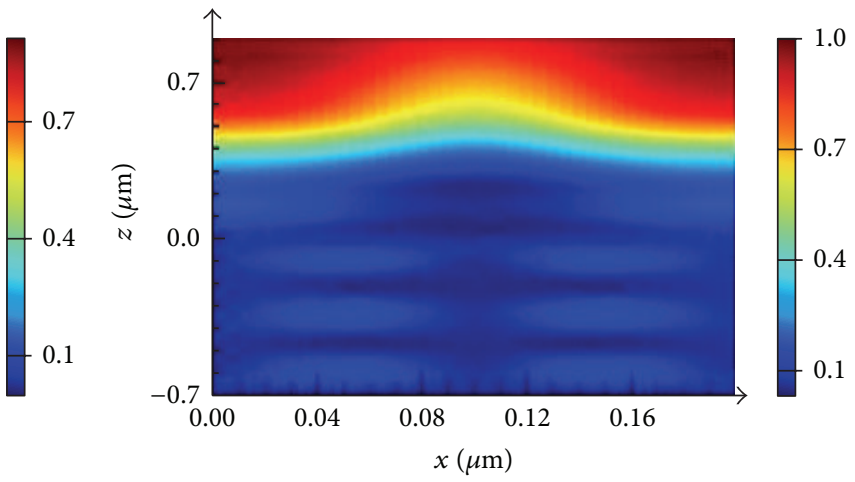

(d)

FIGURE 4: Electric field distribution at $y=0$ plane for different wavelengths. (a) $\lambda=400 \mathrm{~nm}$; (b) $\lambda=500 \mathrm{~nm}$; (c) $\lambda=600 \mathrm{~nm}$; and (d) $\lambda=700 \mathrm{~nm}$. 
down in the case of $\lambda>780 \mathrm{~nm}$, the average absorptivity in the ultrabroadband still remains up to 0.9. Particularly in the violet waveband, it displays extraordinary high absorptive performance. The extremely high absorptivity of the quadrangular frustum pyramid array over the ultrabroad waveband has the significant advantage in comparison with the pure Si nanowires absorber which can only keep high absorptivity in the quite narrow waveband ranging from $300 \mathrm{~nm}$ to $400 \mathrm{~nm}$ [18]. Figures 3(a) and 3(b) show the absorptive spectrum at various incident angles for TE and TM waves, respectively. As can be seen from the figures, the absorptivity keeps nearly unchanged in visible regime when incident angle changes from $30^{\circ}$ to $80^{\circ}$. The absorptivity drops rapidly when the incident angle is $20^{\circ}$ in visible regime. The most important point is that the absorptivity keeps a high level: $>0.7$ in a wide angle range; that is, influence of the absorptivity is insensitive on incident angle in the wide visible regime. To our knowledge, this is the best result in comparison to all the reported absorbers so far.

For the polarization of incident light, it can be seen that the absorptivity has no difference in the same incident light angle. We believe that polarization is unnecessary to be considered here. In microscopic optics, photons with the same energy can excite electron which has the same velocity to conduction band from valence band. In the meanwhile, with the same energy photons excitation, the same heat can be produced. There is no contribution for polarization to the program.

To understand the physical mechanism of the ultrabroad waveband of the quadrangular frustum pyramid array, the electric field distribution for different incident wavelengths is investigated, as shown in Figure 4. In our case, light is mainly absorbed on the top or edge of the quadrangular frustum pyramid. It may be attributed to Fabry-Perot resonance. Light is trapped at the top or edge of the quadrangular frustum pyramid at Fabry-Perot resonance. Light with different wavelengths as different photons energy has different penetration depth. In short wavelength, most of light is absorbed in the quadrangular frustum pyramid array. With increasing of the wavelength, the Si-based substrate begins to contribute to the absorptivity.

\section{Summary}

In summary, we have analyzed a two-dimensional periodic quadrangular frustum pyramid array which can achieve an average absorptivity as high as 0.98 in the whole visible waveband. The absorber can retain very high absorptivity in a wide incident angle range. This high light absorptivity of quadrangular frustum pyramid array is explained as the synergetic effect of Fabry-Perot resonance. With the vast demand of the sustainable and green energy nowadays, we believe that the proposed absorber will find its application in those areas related to solar energy harvesting.

\section{Conflict of Interests}

The authors declare that there is no conflict of interests regarding the publication of this paper.

\section{References}

[1] L. He, C. Jiang, D. Rusli, and H. Wang, "Highly efficient Si-nanorods/organic hybrid core-sheath heterojunction solar cells," Applied Physics Letters, vol. 99, Article ID 021104, 2011.

[2] O. L. Muskens, J. G. Rivas, R. E. Algra, E. P. A. M. Bakkers, and A. Lagendijk, "Design of light scattering in nanowire materials for photovoltaic applications," Nano Letters, vol. 8, no. 9, pp. 2638-2642, 2008.

[3] J. Zhu, Z. Yu, G. F. Burkhart et al., "Optical absorption enhancement in amorphous silicon nanowire and nanocone arrays," Nano Letters, vol. 9, no. 1, pp. 279-282, 2009.

[4] H. Fang, X. Li, S. Song, Y. Xu, and J. Zhu, "Fabrication of slantingly-aligned silicon nanowire arrays for solar cell applications," Nanotechnology, vol. 19, Article ID 255703, 2008.

[5] M. D. Kelzenberg, D. B. Turner-Evans, B. M. Kayes et al., "Photovoltaic measurements in single-nanowire silicon solar cells," Nano Letters, vol. 8, no. 2, pp. 710-714, 2008.

[6] T. Stelzner, M. Pietsch, G. Andrä, F. Falk, E. Ose, and S. Christiansen, "Silicon nanowire-based solar cells," Nanotechnology, vol. 19, no. 29, Article ID 295203, 2008.

[7] J. Li, H. Yu, S. Wong et al., "Si nanopillar array optimization on Si thin films for solar energy harvesting," Applied Physics Letters, vol. 95, Article ID 033102, 2009.

[8] Y.-F. Huang, S. Chattopadhyay, Y.-J. Jen et al., "Improved broadband and quasi-omnidirectional anti-reflection properties with biomimetic silicon nanostructures," Nature Nanotechnology, vol. 2, no. 12, pp. 770-774, 2007.

[9] S. E. Han and G. Chen, "Optical absorption enhancement in silicon nanohole arrays for solar photovoltaics," Nano Letters, vol. 10, no. 3, pp. 1012-1015, 2010.

[10] C. Lin and M. L. Povinelli, "Optical absorption enhancement in silicon nanowire arrays with a large lattice constant for photovoltaic applications," Optics Express, vol. 17, no. 22, pp. 19371-19381, 2009.

[11] H.-Y. Tseng, C.-K. Lee, S.-Y. Wu et al., "Au nanorings for enhancing absorption and backscattering monitored with optical coherence tomography," Nanotechnology, vol. 21, no. 29, Article ID 295102, 2010.

[12] B. C. P. Sturmberg, K. B. Dossou, L. C. Botten et al., "Modal analysis of enhanced absorption in silicon nanowire arrays," Optics Express, vol. 19, no. 19, pp. A1067-A1081, 2011.

[13] Y. Cui, J. Xu, K. Fung et al., "Ultrabroadband light absorption by a sawtooth anisotropic metamaterial slab," Nano Letters, vol. 12, no. 3, pp. 1443-1447, 2012.

[14] Q. Liang, T. Wang, Z. Lu, Q. Sun, Y. Fu, and W. Yu, "Metamaterial-based two dimensional plasmonic subwavelength structures offer the broadest waveband light harvesting," Advanced Optical Materials, vol. 1, pp. 43-49, 2013.

[15] S. Cao, W. Yu, T. Wang et al., "Two-dimensional subwavelength meta-nanopillar array for efficient visible light absorption," Applied Physics Letters, vol. 102, Article ID 161109, 2013.

[16] U. Guler, A. Boltasseva, and V. M. Shalaev, "Refractory plasmonics," Science, vol. 344, no. 6181, pp. 263-264, 2014.

[17] M. B. Dühring and O. Sigmund, "Optimization of extraordinary optical absorption in plasmonic and dielectric structures," Journal of the Optical Society of America B, vol. 30, no. 5, pp. 1154-1160, 2013.

[18] L. Hu and G. Chen, "Analysis of optical absorption in silicon nanowire arrays for photovoltaic applications," Nano Letters, vol. 7, no. 11, pp. 3249-3252, 2007. 

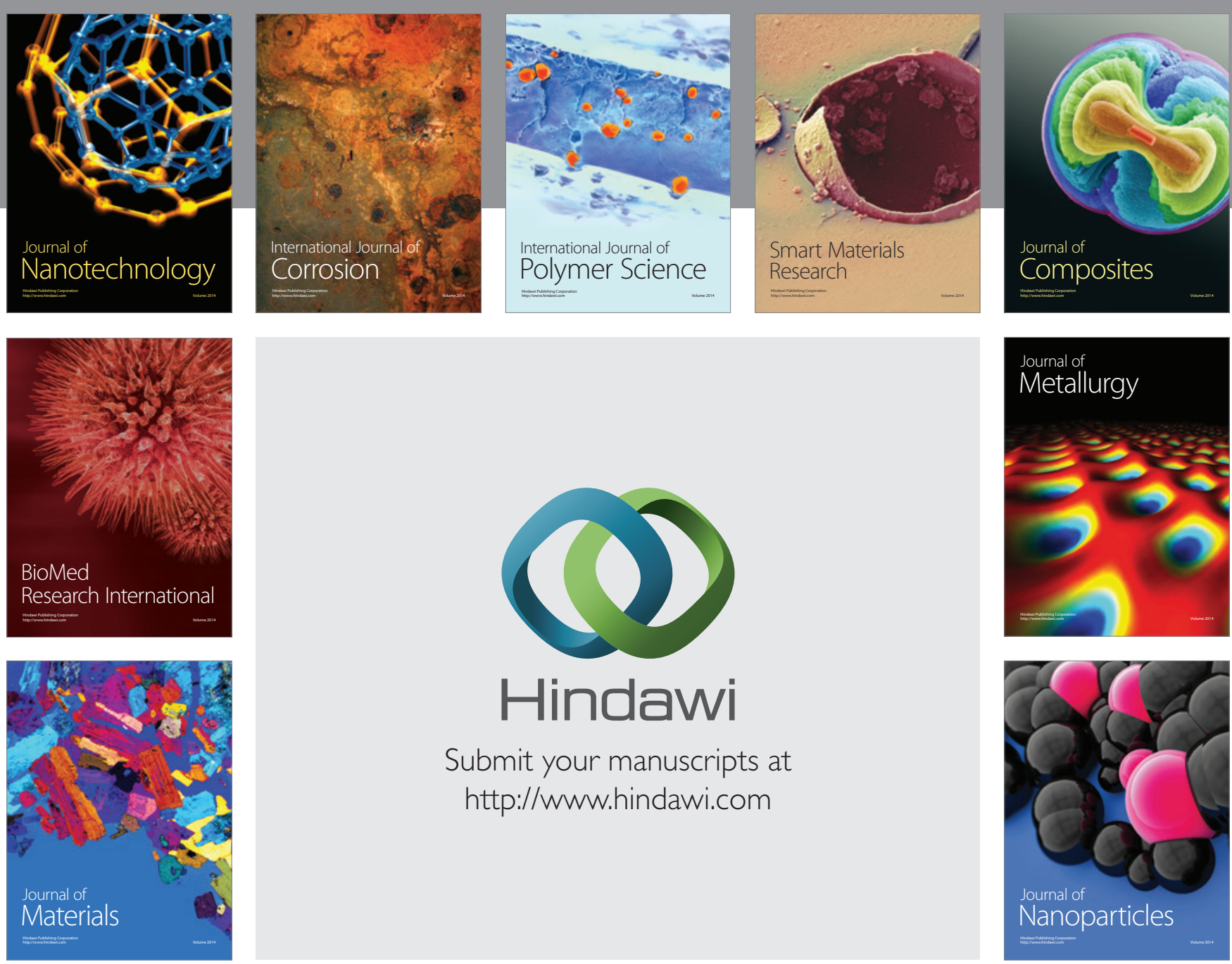

Submit your manuscripts at http://www.hindawi.com
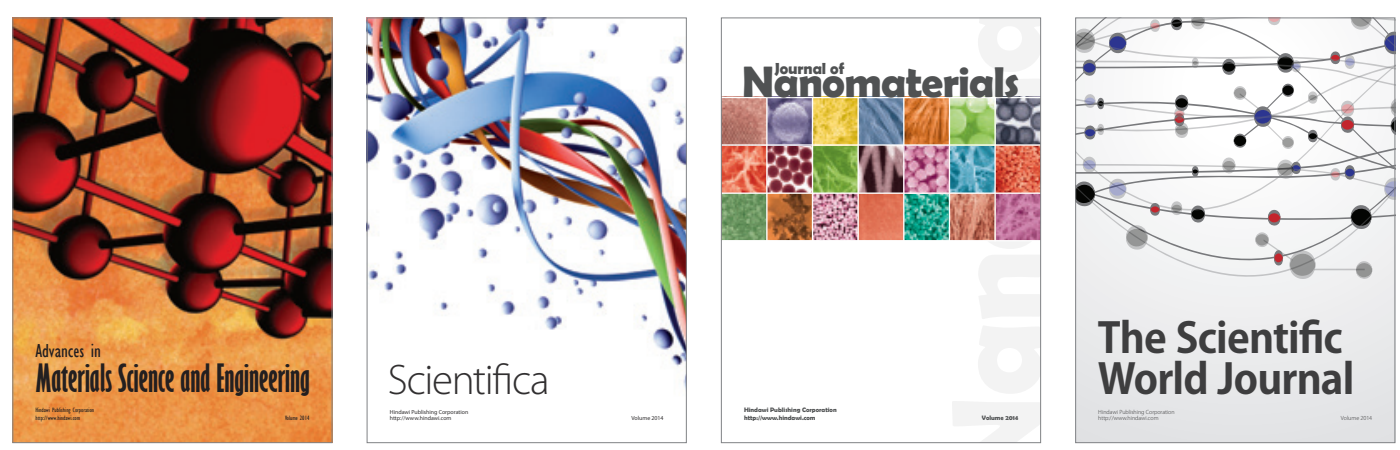

\section{The Scientific World Journal}
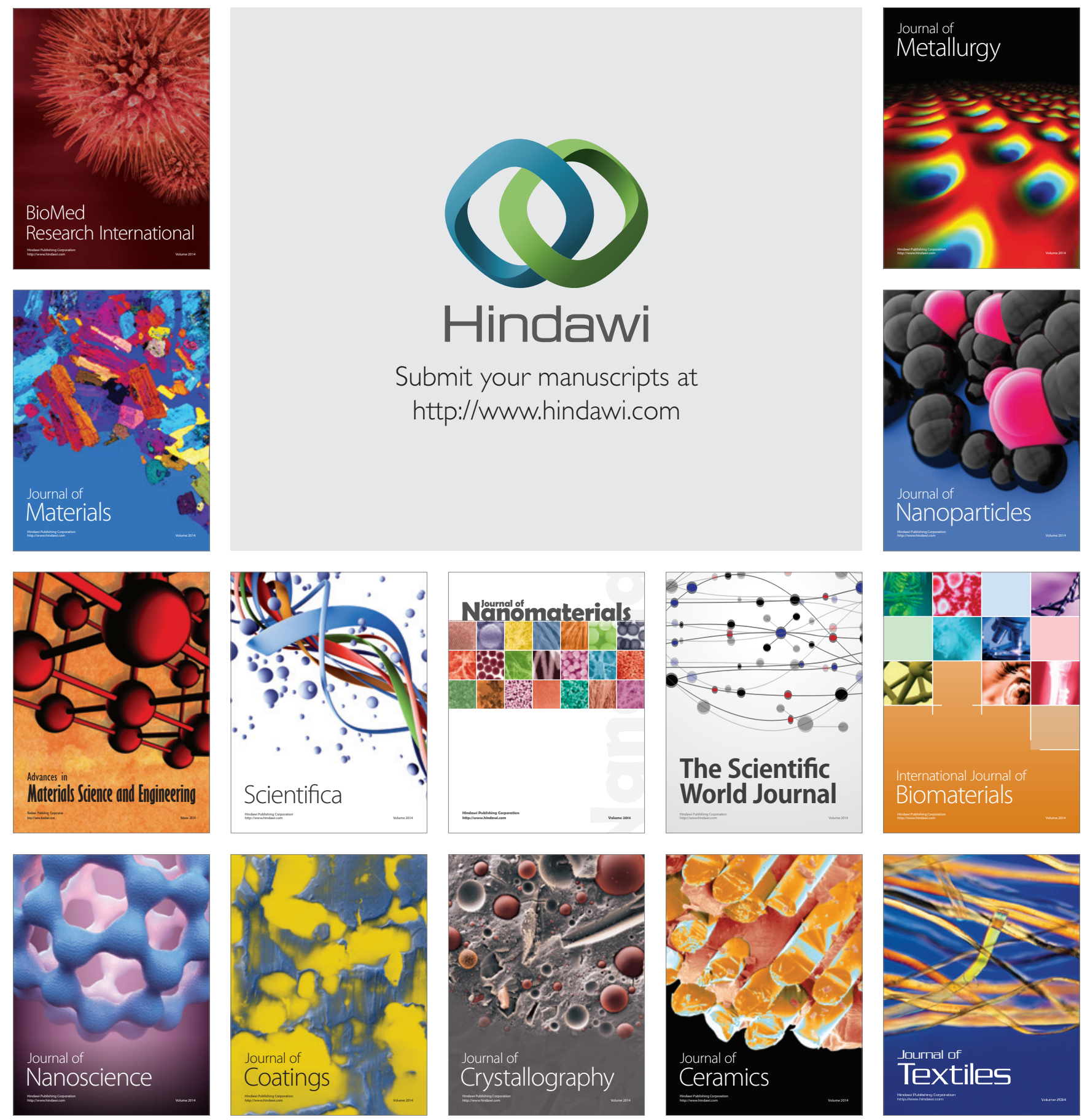